\title{
Comunicarse en la escuela plurilingüe: la formación en lengua extranjera del futuro profesorado
}

\author{
Communicating in the multilingual school: training in a foreign \\ language for future teachers
}

Ester Trigo Ibáñez. Universidad de Cádiz. España.

ester.trigo@uca.es

$[\underline{\mathrm{CV}}] \mathbf{C} \mathrm{R}^{\mathrm{O}} \mathrm{O}$

Inmaculada Clotilde Santos Díaz. Universidad de Málaga. España.

santosdiaz@uma.es

$[\mathrm{CV}](1) \mathrm{G} \mathrm{R}^{\mathrm{g}} \mathrm{C}$

Guillermina Jiménez López. Universidad de Málaga. España.

gjimenez@uma.es

$[\underline{\mathrm{CV}}] \bigcirc \mathrm{R}^{\mathrm{a}}$

Cómo citar este artículo / Referencia normalizada

Trigo, E., Santos Díaz, I. C. y Jiménez López, G. (2021). Comunicarse en la escuela plurilingüe:

la formación en lengua extranjera del futuro profesorado. Revista Latina de Comunicación Social,

79, 53-75. https://www.doi.org/10.4185/RLCS-2021-1497

\section{RESUMEN}

Introducción: La necesidad de comunicación en lengua extranjera de la sociedad actual se manifiesta en las políticas educativas que promueven una enseñanza plurilingüe. Este estudio se realizó con el propósito de indagar en la formación en lengua extranjera de los futuros docentes. Metodología: Se diseñó un cuestionario con preguntas sociodemográficas y relacionadas con el aprendizaje de una lengua y se aplicaron unas pruebas de nivel de Cambridge. La muestra de estudio está formada por 427 estudiantes del Grado en Educación Primaria de la Universidad de Málaga durante el curso 2018/2019. El análisis de los datos se realizó con el paquete estadístico SPSS (v. 22). Resultados: Se ha constatado que un $48,01 \%$ de los informantes cursan estudios de idiomas al margen de la universidad, un 86,48 \% no ha realizado ningún curso o estancia en el extranjero, un 99,06\% tiene acreditado algún nivel de inglés y la segunda lengua extranjera para la mayoría es el francés. En cuanto a la relación de estas variables con la puntuación obtenida en las pruebas de comprensión oral y escrita, en todos los casos son significativas. Discusión: Existe una divergencia entre el nivel alcanzado en las pruebas y el nivel acreditado y exigido como requisito mínimo por las administraciones públicas. Conclusiones: Estos hallazgos son de gran relevancia para conocer la formación linguiística de un colectivo que juega un papel esencial en la enseñanza bilingüe y para establecer líneas de actuación futuras con objeto de promover la mejora de la competencia en lengua extranjera. 
PALABRAS CLAVE: formación del profesorado; enseñanza de una lengua; inglés como lengua extranjera; comprensión lectora; comprensión oral; bilingüismo.

\begin{abstract}
Introduction: The need for communication in a foreign language today is manifested in educational policies that promote multilingual teaching. This study was carried out with the purpose of investigating the foreign language training of future teachers. Methodology: A questionnaire was designed with sociodemographic questions and questions related to learning a language, and Cambridge level tests were applied. The study sample is made up of 427 students of the Degree in Primary Education at the University of Malaga during the 2018/2019 academic year. Data analysis was performed with the SPSS statistical package (v. 22). Results: It has been found that $48.01 \%$ of the informants' study languages outside the university, $86.48 \%$ have not taken any course or stay abroad, 99.06\% have accredited some level of English and the second foreign language for most is French. Regarding the relationship of these variables with the score obtained in the oral and written comprehension tests, in all cases they are significant. Discussion: There is a divergence between the level achieved in the tests and the level accredited and required as a minimum requirement by public administrations. Conclusions: These findings are of great relevance to know the linguistic formation of a group that plays an essential role in bilingual education and to establish future lines of action in order to promote the improvement of foreign language competence.
\end{abstract}

KEYWORDS: teacher training; teaching a language; English as a foreign language; reading comprehension; oral comprehension; bilingualism.

\title{
CONTENIDO
}

1. Introducción. 2. Objetivos. 3. Metodología. 4. Discusión/Resultados. 5. Conclusiones. 6. Bibliografía

\section{Introducción}

En la sociedad actual resulta indispensable saber comunicarse en más de una lengua pues esta habilidad nos abre puertas hacia otras culturas, propicia la adquisición de conocimiento, contribuye a ampliar las fronteras en busca de amistades, formas de ocio, oportunidades de empleo o estilos de vida. Esta creencia parece estar muy extendida en el mundo hiperconectado y globalizado en el que vivimos (Santos-Díaz et ál., 2020; Shafirova et ál., 2020; Wulz, 2015; Zhang \& Cassany, 2019).

Sin embargo, una rápida mirada a los resultados educativos manifiestos en pruebas externas deja entrever una amplia lista de tareas pendientes en lo que respecta al desarrollo de las competencias comunicativas de nuestros estudiantes, en general (Lorenzo, 2016; Pérez Invernón, 2019; Trigo et ál., 2019), y a su manejo de lenguas extranjeras, en particular (Lorenzo \& Rodríguez, 2014; Pérez Invernón et ál., 2016; Pavón \& Pérez Invernón, 2017).

Habida cuenta de esta problemática, las instituciones competentes en educación han ido desarrollando estrategias encaminadas al establecimiento paulatino de un modelo de escuela plurilingüe, capaz de responder a los retos que la sociedad del conocimiento demanda (Abdelillah-Bauer, 2011; Coyle, 2015; Lauridesen \& Lilemose, 2015; Meyer et ál., 2015; Mottareale, 2017). No obstante, como apuntan Guardamillas \& Alcaraz (2017, p. 97) en los distintos territorios españoles existe "un significativo grado de heterogeneidad tanto en los requisitos lingüísticos y metodológicos del profesorado como en las asignaturas incluidas en los planes de estudio bilingües". En cuanto al requisito lingüístico, a excepción de Navarra y Madrid que demandan un nivel de C1, todas las Comunidades Autónomas 
exigen un nivel de acreditación lingüística de B2 según el Marco Común Europeo de Referencia de las Lenguas, en adelante, MCERL (Consejo de Europa, 2001). Sin embargo, en el ámbito metodológico se da una mayor disparidad, dividida en tres grupos:

Aquellas comunidades que no exigen formación metodológica para impartir docencia en áreas no lingüísticas en lengua extranjera. En este grupo se encuentran Madrid, Castilla y León, Cataluña y Valencia.

- Aquellas que mencionan la formación metodológica, pero no exigen de forma explícita la centrada en el enfoque AICLE. Se sitúan en este grupo Andalucía y Extremadura.

- Aquellas que exigen un número de horas de formación permanente en el enfoque AICLE. Se incluyen en este grupo Canarias y País Vasco.

En este sentido, centrándonos en Andalucía, contexto en el que se enmarca esta investigación, con el objetivo de adaptar la sociedad andaluza a los nuevos retos que plantea el fenómeno de la globalización y promover el aprendizaje de idiomas y la diversidad lingüística, la Junta de Andalucía aprobó en el año 2005 el Plan de Fomento del Plurilingüismo (Acuerdo de 22 de marzo de 2005 del Consejo de Gobierno) y en 2017 el Plan Estratégico de Desarrollo de las Lenguas en Andalucía (Acuerdo de 24 de enero de 2017, del Consejo de Gobierno). De esta forma, se deja patente que, según se vayan afianzando los programas bilingües y aumente el nivel de competencia entre el alumnado y el profesorado, en el futuro, será requisito indispensable para impartir docencia en el programa bilingüe tener un nivel de C1, para lo cual se contará con la colaboración de las Escuelas Oficiales de Idiomas (EEOOII) y los Centros de Formación Permanente del Profesorado (CEP) para garantizar la paulatina acreditación lingüística y capacitación metodológica del profesorado.

En consonancia con lo expuesto anteriormente, se hace necesario prestar atención a los procesos de formación del profesorado:

- Por un lado, en el marco de la formación permanente, será preciso analizar las políticas implementadas por las distintas Comunidades Autónomas (Guarro et ál., 2017) y reflexionar sobre el efecto que esta formación ejerce sobre el desarrollo profesional de los docentes (Álvarez \& Alejaldre, 2019; Forteza et ál., 2020; González-Peiteado, 2013; Sanz Trigueros, \& Guillén, 2016) y en el rendimiento del alumnado (Cordero, \& Gil-Izquierdo, 2018; Gómez López, 2014).

- Por otro lado, en el ámbito de la formación inicial de docentes, sería conveniente reflexionar sobre la concepción que los futuros docentes poseen acerca de su proyección profesional (Martínez-Agudo, 2011) así como propiciar procesos de meta-reflexión (Castillo et ál., 2020; Estrada \& Zayas, 2019; Fernández-Viciana \& Fernández-Costales, 2019; Sologuren \& Castillo-Fadić, 2020; Torrado-Crespón, 2018), lo que les hará más conscientes de cómo se aprende y, por tanto, les pondrá en mejor disposición para enseñar.

De acuerdo con estos planteamientos, si bien el perfil del profesor idóneo en lengua extranjera ha sido ampliamente estudiado (Martínez-Agudo, 2001; Yance et ál., 2011; Martín del Pozo, 2013; Páez, 2013), consideramos que existen aspectos que no se han tenido en cuenta y que resultan fundamentales para realizar una efectiva labor docente. Todas las investigaciones se centran en la competencia lingüística y metodológica e incluyen una dimensión centrada en la personalidad del profesorado, pero no atienden, por ejemplo, a la formación de lectores para una escuela plurilingüe (Gómez López, 2014; Fernández-Ruiz et ál., 2018; Pérez-Parejo et ál., 2018; Rivera \& Romero, 2020) ni a las estrategias que, desde la formación inicial, pueden desarrollarse para mejorar la capacitación docente y garantizar la egresión con éxito en las condiciones que demanda la escuela actual (Delicado \& Pavón, 2015). Dentro de estas estrategias debería tenerse en cuenta el impacto que ejercen las estancias realizadas por los futuros docentes durante su formación (Choudaha, 2014; Sanz-de-la-Cal, 2014; Santos-Díaz, 2015; Sanz-Trigueros \& Guillén, 2020). 
Con respecto a la competencia léxica en lengua extranjera, Santos-Díaz (2015) analiza el impacto de las estancias en el extranjero del futuro profesorado de Educación Secundaria. Para ello, analiza el número de palabras escritas en una prueba de disponibilidad léxica en inglés y francés y distingue entre tres tipos de estancia: con la beca Erasmus, estudios con otro tipo de beca (usualmente con una duración de un mes en el periodo estival) y otras estancias sin becas. El estudio concluye que, si bien todos los informantes que han realizado algún tipo de estancia escriben un mayor número de palabras en lengua extranjera que los que no han realizado ninguna, la producción léxica mayor se da cuando los informantes han realizado una estancia Erasmus, seguida de otros estudios becados. Estos hallazgos son corroborados por Herranz \& Marcos (2019) y Šifrar (2020).

Con respecto a la formación metodológica, Sanz-de-la-Cal (2014) y Sanz-Trigueros \& Guillén (2020) señalan que la posibilidad de realizar prácticas en otros países hace que los futuros docentes puedan vivir en inmersión y aprender del modus docendi no solo de otros profesores experimentados, sino también de la forma de plantear la enseñanza según las políticas educativas de los distintos países (Wulz, 2015).

Habida cuenta de la necesidad de perfilar de una forma más completa las cualidades inherentes a todo buen docente y de solucionar los aspectos deficitarios, en este trabajo nos centramos en la formación inicial de docentes. Así, analizaremos el dominio que los estudiantes tienen en inglés como lengua extranjera y los factores que influyen en la mejora de sus competencias profesionales para ejercer la docencia dentro de la actual escuela plurilingüe.

\section{Objetivos}

Este estudio se plantea dos objetivos complementarios. Por un lado, conocer la formación en lengua extranjera de estudiantes del Grado en Educación Primaria a través de variables como el nivel de inglés acreditado oficialmente, el conocimiento de otros idiomas, la realización de estancias en el extranjero y los estudios al margen de la universidad. Por otro lado, pretende analizar la relación de esas variables con los resultados obtenidos en una prueba de nivel de Cambridge y, más específicamente, en las destrezas de Listening, Use of English y Reading.

\section{Metodología}

Este trabajo se enmarca en el paradigma cuantitativo y posee un diseño experimental pues trata de corroborar la relación de las variables establecidas con el domino de la lengua inglesa demostrado al realizar una prueba (Sáez, 2017). En primera instancia, se realiza una investigación descriptiva ya que, a través de un cuestionario, se analiza la formación en lengua extranjera que poseen los sujetos investigados (McMillan \& Schumacher, 2006). En segunda instancia, se procede a realizar una triangulación al contrastarse los hallazgos con los resultados obtenidos por los informantes en la prueba de nivel de Cambridge y poder verificar la información (Aguilar \& Barroso, 2015).

\subsection{Participantes}

La muestra está formada por un total de 427 estudiantes que cursaban el Grado en Educación Primaria en la Universidad de Málaga durante el curso 2018/2019. Su edad oscila entre los 18 y los 49 años ( $\bar{x}=$ $20,51 ; \sigma=3,356)$. Existe un perfil predominantemente femenino donde el $73,07 \%$ son mujeres $(n=312)$ frente al $26,93 \%(n=115)$ que son hombres. Los estudiantes pertenecen a los cuatro cursos atendiendo a la siguiente distribución: en primero 108 estudiantes $(25,29 \%)$, en segundo 110 estudiantes 
RLCS, Revista Latina de Comunicación Social, 79, 53-75

\begin{tabular}{ll|l|l|l} 
[Investigación] 10.4185/RLCS-2021-1497 & ISSN 1138-5820 & Año 2021
\end{tabular}

$(25,76 \%)$, en tercero 182 estudiantes $(42,62 \%)$ y en cuarto $27(6,32 \%)$. De esta forma, el error de muestreo se sitúa en un 6,2\% sobre la población total de alumnado matriculado $(\mathrm{N}=1100)$.

\subsection{Instrumento}

La presente investigación se ha llevado a cabo utilizando dos instrumentos de recogida de datos. El primer instrumento consiste en un cuestionario inédito que consta de 31 preguntas, 23 cerradas y 8 abiertas (Martín-Izard, 2010). Además de solicitar información sociodemográfica como el sexo y la edad, se incluyen otras que indagan sobre el nivel de conocimiento de la lengua extranjera, la frecuencia de uso e importancia que se le otorga a la lengua extranjera en las distintas asignaturas, la capacitación y nivel de formación adquiridos en cada una de las destrezas lingüísticas para su enseñanza, entre otras variables. La validación del cuestionario se ha llevado a cabo en dos fases:

a) En primer lugar, siguiendo las pautas marcadas por Cabero \& Llorente (2013); Escobar \& Cuervo (2008) y Robles \& Rojas (2015), el cuestionario se sometió a una fase de valoración externa en la que estuvieron implicados jueces expertos de diferentes universidades (Universidad de Málaga, de Granada, de Cádiz y la Universidad Internacional de La Rioja). El medio de contacto utilizado fue el correo electrónico, donde se incluyó una carta de presentación en la que se explicaba la naturaleza del estudio, la petición de colaboración para la validación, el cuestionario en sí y las tablas para la valoración acerca de la pertinencia y la comprensión de cada pregunta utilizando una escala tipo Likert de 5 valores (Matas, 2018). La valoración general de los expertos fue muy positiva, consideraron el instrumento muy relevante y adecuado para el objeto de estudio de la investigación y realizaron algunas sugerencias de mejora que se incorporaron en el cuestionario. Las pruebas de fiabilidad estadística del instrumento confirmaron un Alpha de Cronbach de 0,982, lo que posibilitará la utilización del cuestionario en investigaciones posteriores.

b) A continuación, se realizó una fase de pilotaje manteniendo los principios de Dörnyei \& Taguchi (2010) en el que marcamos las preguntas que se consideraron innecesarias y cualquier cambio que pudiera mejorar el cuestionario. Para ello, se eligió una muestra de 105 alumnos y alumnas de los 120 matriculados en los grupos C y E -70 en el grupo C y 50 en el E- de cuarto curso del Grado en Educación Primaria del año académico 2017/18. El tamaño de la muestra para el pilotaje se ajusta a la descripción de Dörnyei \& Taguchi (2010), debe ser de aproximadamente 100 participantes (por motivos estadísticos no menos de 50). Ya que esta cantidad de informantes permite al investigador un análisis significativo que encamina el cuestionario hacia su versión final.

El segundo instrumento, se trata de una prueba estandarizada para evaluar el nivel en inglés en las distintas destrezas facilitadas por la corporación Cambridge (Cambridge English Language Assessment, 2016) y que no están publicadas, para evitar que el alumnado las conozca previamente. Concretamente se han evaluado los siguientes aspectos de la lengua:

a) Reading and Use of English: se estructura en siete partes con un total de 52 preguntas divididas en dos segmentos principales que se distribuyen de acuerdo con los conocimientos que evalúan. La comprensión lectora o Reading contiene las tareas 1, 5, 6 y 7; el segmento Use of English acoge los ejercicios del 2 al 4 que describen el nivel de conocimientos gramaticales y de vocabulario. El rango de puntuación de cada actividad por cada respuesta correcta oscila entre un punto para los ejercicios 1, 2, 3 y 7; mientras que las tareas 4, 5 y 6 otorgan dos puntos. La puntuación máxima para el Reading es 42 y para el Use of English 28. Las tareas correspondientes al Reading exponen una variada gama de textos de distinta longitud en los que los participantes tendrán que demostrar sus destrezas para comprender información general y específica, opiniones, actitudes, propósitos, etc. En cuanto a las actividades del Use of 
English, tienen como objetivo evaluar si los estudiantes poseen un vocabulario variado relacionado con diversos temas y diferentes estructuras gramaticales.

b) Listening: esta prueba de comprensión oral consta de cuatro partes con un total de 30 preguntas y contiene la grabación de un texto o varios textos de diferente tipología como monólogos, diálogos, entrevistas, programas de radio, noticias, etc. Los participantes después de oír dos veces la grabación deben contestar preguntas de elección múltiple, de completar frases y unión múltiple. Cada una de las respuestas correctas se valorará con un punto, pudiendo obtener una puntuación máxima de 30 puntos.

En cuanto a la equivalencia de los resultados obtenidos con los niveles del MCERL, Cambridge English Language Assessment (2016) incluye un conversor que resumimos en la Tabla 1. La puntuación en la que no aparece ningún nivel se ha codificado como inferior a B1 ya que el conversor no distingue a partir de ese nivel:

Tabla 1. Conversor de puntuaciones en los test de Cambridge a los niveles del MCERL.

\begin{tabular}{lll}
\hline Test & Puntuación & Nivel del MCERL \\
Reading & 37 & $\mathrm{C} 1$ \\
& 24 & $\mathrm{~B} 2$ \\
& 16 & $\mathrm{~B} 1$ \\
Use of English & 10 & - \\
& 24 & $\mathrm{C} 1$ \\
& 18 & $\mathrm{~B} 2$ \\
Listening & 11 & $\mathrm{~B} 1$ \\
& 7 & - \\
& 27 & $\mathrm{C} 1$ \\
& 18 & $\mathrm{~B} 2$ \\
& 12 & $\mathrm{~B} 1$ \\
\hline
\end{tabular}

Fuente: Elaboración propia.

\subsection{Procedimiento}

El proceso de aplicación de los instrumentos, acceso a los informantes y recogida de datos se distribuyó en cuatro fases. En una primera fase, se solicitó colaboración tanto al Vicedecanato de estudiantes y movilidad de la Facultad de Ciencias de la Educación de la Universidad de Málaga, como al profesorado del Grado en Educación Primaria para explicar la naturaleza de la investigación, la confidencialidad de los datos recabados y solicitar su colaboración.

En una segunda fase, previa firma de un consentimiento informado, se pasó el cuestionario y la prueba de nivel al alumnado. Ambas pruebas se realizaron en papel de forma presencial durante el curso 2018/2019 en horario lectivo. Debido a la duración de las pruebas de Cambridge, se tuvieron que realizar en momentos diferentes: un primer día dedicado al cuestionario y a las pruebas de Reading y Use of English, un segundo día para el Listening y Writing y, un tercer día, para un grupo más reducido de voluntarios para el Speaking.

En una tercera fase, se corrigieron las pruebas de nivel y se enviaron las puntuaciones obtenidas a cada profesor para su difusión entre los participantes. A continuación, se volcaron las respuestas en una matriz en Excel y, posteriormente, se trasladaron al programa estadístico SPSS (v. 22) para su procesamiento estadístico. 
En una cuarta fase, se realizaron análisis descriptivos (como el cálculo de medias y distribución de frecuencias) e inferenciales a través del contraste de hipótesis con t de Student. Las variables objeto de análisis fueron de dos tipos. Por un lado, cuatro correspondientes al cuestionario referidas al conocimiento y formación en lengua extranjera: 1) Estudio de una lengua extranjera, 2) Estancias en el extranjero, 3) Nivel acreditado en inglés y 4) Conocimiento de otras lenguas. Por otro lado, las variables que miden las destrezas evaluadas con la prueba de Cambridge: 1) Reading, 2) Use of English y 3) Listening. Estas variables se han analizado tomando la puntuación exacta obtenida, así como utilizando la conversión de sus resultados según los niveles del MCERL (vid. Tabla 1).

\section{Resultados}

\subsection{Formación en lengua extranjera}

Para conocer el tipo de formación recibida sobre la lengua inglesa, se solicitó información sobre su estudio en España, con indicación del lugar donde lo hace, así como de las estancias en el extranjero. Ambas modalidades de formación no son excluyentes, ya que la mayoría de los estudiantes que realizan estancias en el extranjero lo hacen para perfeccionar el idioma que suelen estudiar en España. Cabe mencionar que en el Grado de Educación Primaria las asignaturas específicas que se imparten en la mención de inglés o en el grupo bilingüe, si bien usan este idioma como lengua de instrucción, tienen como objetivo enseñar aspectos metodológicos y no lingüísticos.

\subsubsection{Estudio de una lengua extranjera}

En primer lugar, los estudiantes tenían que contestar a la siguiente pregunta “¿Estás estudiando inglés al margen de tus estudios de Grado?" Casi la mitad de los alumnos estudia una lengua extranjera, concretamente un 47,54\%. Un 43,33\% estudia únicamente inglés ( $\mathrm{n}=185)$, un $0,94 \%$ inglés y otras lenguas $(n=4)$ y un $3,28 \%$ otras lenguas excluyendo inglés $(n=14)$.

A continuación, quienes habían contestado afirmativamente a la pregunta anterior, tenían que indicar el nombre del centro o institución. Una vez recopilada la información, las respuestas se agruparon según el tipo de institución o centro - vid. Figura 1-. Por orden decreciente de elección, se sitúan las academias $(35,60 \%)$, la Escuela Oficial de Idiomas (6,79\%), las clases particulares $(2,81 \%)$, las clases en la Fundación General de la Universidad de Málaga (1,87 \%) y tan solo un informante eligió el Centro de Arte y Cultura del Ayuntamiento $(0,23 \%)$ y otro las clases con un profesor nativo $(0,23 \%)$. A pesar de que las clases con un profesor nativo podrían considerarse también clases particulares, se ha dejado constancia de forma diferenciada para poner en valor la respuesta del estudiante que quería marcar la diferencia en cuanto a las características de su profesor de inglés. 


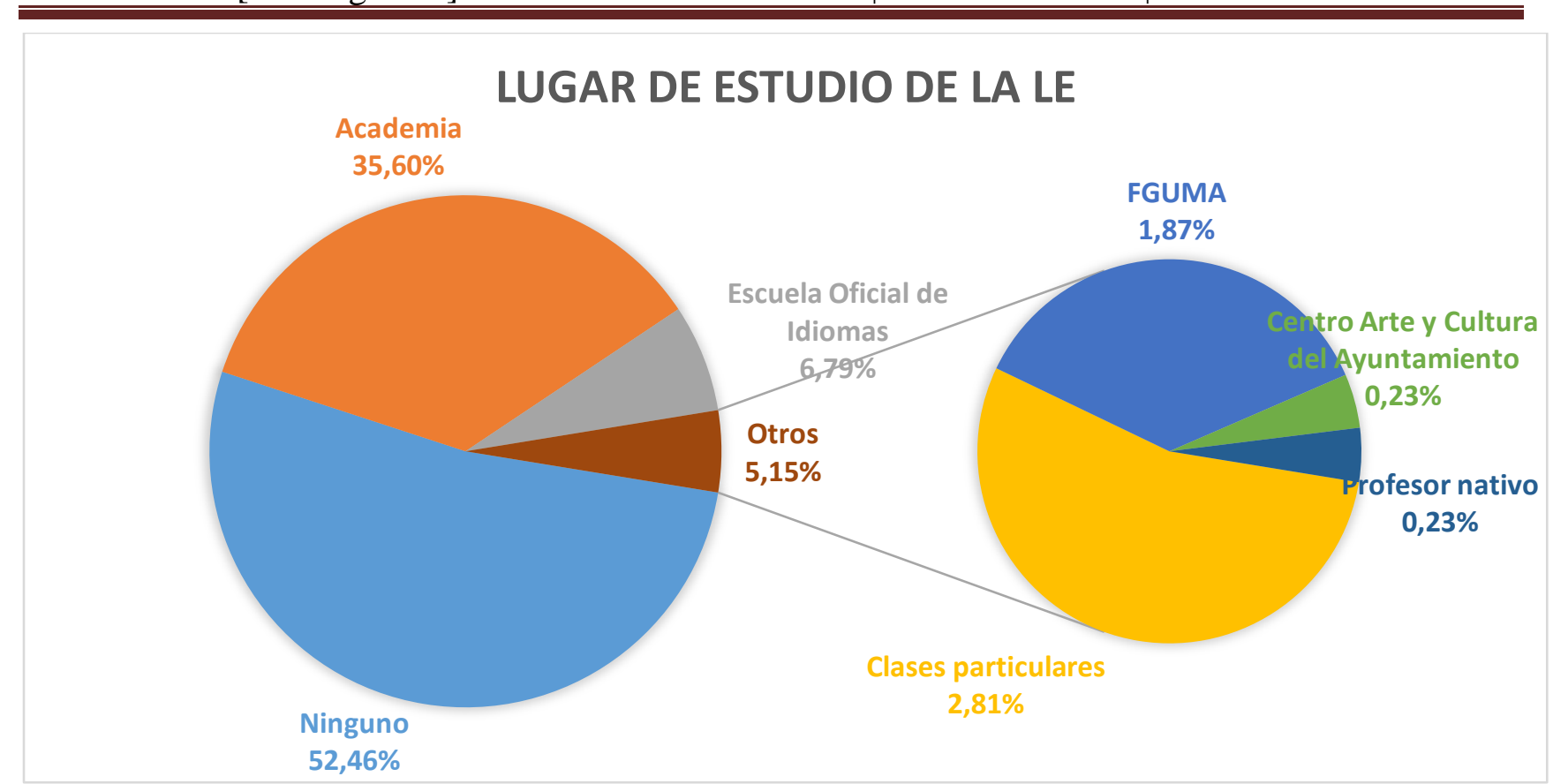

Figura 1. Lugar de estudio de la lengua extranjera.

Fuente: Elaboración propia.

\subsubsection{Realización de estancias en el extranjero}

Esta pregunta supone un acercamiento a la movilidad internacional de los estudiantes, con independencia del tipo de estancia que hayan realizado en el extranjero. Tan solo un 15,69\% (n=67) de los estudiantes ha realizado algún curso o estancia en el extranjero frente al 84,31\% ( $n=360$ ) que no ha realizado ninguno.

\subsection{Nivel en las destrezas}

\subsubsection{Resultados obtenidos en las pruebas}

Una vez corregidas las pruebas de inglés, obtuvimos una nota numérica que nos permitió agrupar a los informantes por el nivel alcanzado según el MCERL. La puntuación media obtenida en cada prueba ha sido 16,80 en el Reading, 10,47 en el Use of English y 11,34 en el Listening. Tan solo la puntuación en el Reading es equivalente a un nivel B1 mientras que en las otras dos pruebas se correspondiente con un nivel inferior a B1.

La Figura 2 muestra los resultados en las tres pruebas. Se ha usado la misma nomenclatura para referirse al tipo de destreza que aparece en la prueba de Cambridge. La distribución de los resultados muestra que en torno a la mitad de los estudiantes ha conseguido un nivel B1, que será el exigido para poder obtener el título de Graduado en Educación Primaria ya que en la gran mayoría suele ser el idioma elegido para la acreditación de este requisito. Concretamente han alcanzado un B1 o un nivel superior un 53,16 \% en el Reading, un 51,29\% en el Use of English y un 46,14\% en el Listening. En cuanto a las pruebas que han obtenido resultados inferiores de un nivel B1, por orden de mayor a menor son el Listening (53,86 \%), Use of English (48,71\%) y el Reading (46,84\%). En todos los casos, el porcentaje de los estudiantes que ha alcanzado un nivel C1 es muy reducido siendo superior en el Use of English (3,28 \%), seguido del Listening (2,81\%) y el Reading (1,87\%). 


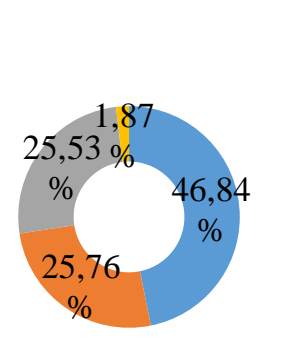

Inferior a

B1

Nivel B1

Nivel B2

Nivel C1

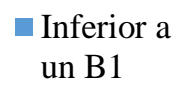

Nivel B2

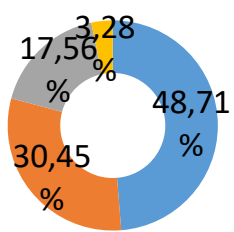

Nivel C1
Inferior a

B1

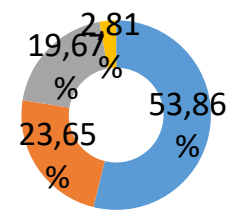

Nivel B1

Nivel B2

Nivel C1

Figura 2. Diagrama de sectores del nivel alcanzado según el MCERL en la prueba de comprensión escrita.

Fuente: Elaboración propia.

\subsubsection{Nivel acreditado}

Teniendo en cuenta que algunos certificados de nivel incluyen un desglose por separado según las destrezas, los informantes pueden afirmar que han adquirido más o menos nivel según el tipo de destreza. Casi todos los informantes afirman tener acreditado algún nivel de inglés, concretamente el $99,06 \%(\mathrm{n}=423)$ frente al 0,94 \% $(\mathrm{n}=4)$ que no lo tiene. Como muestra la Figura 3, en la única destreza que un informante afirma tener un $\mathrm{C} 2$ es en el Reading. La mayor parte de las respuestas se concentran en un nivel B1, seguido de un nivel B2, un nivel A2, un nivel C1 y un nivel A1.

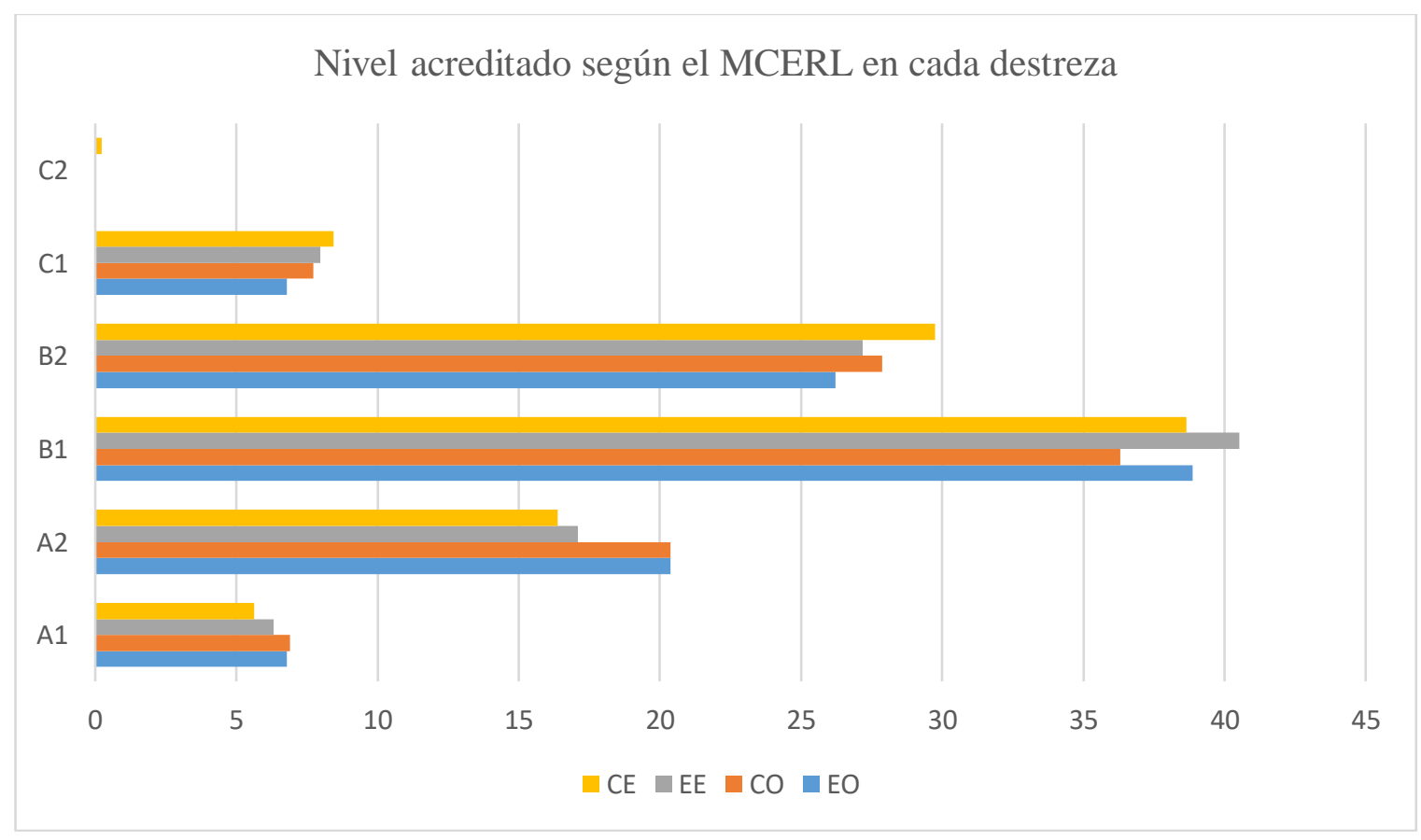

Figura 3. Niveles acreditados en inglés según destrezas.

Fuente: Elaboración propia. 
Con respecto al dominio de otras lenguas, el francés es la segunda lengua que los estudiantes afirman tener acreditado algún nivel, concretamente un $64,40 \%(n=275)$ frente a un 35,60 \% $(n=152)$ que no tiene acreditado ningún nivel. El resto de las lenguas que los informantes han contestado afirmativamente a la pregunta son por orden descendente: el italiano $(n=13)$, alemán $(n=8)$ y hay tan solo un informante que afirma conocer otro de los siguientes idiomas, en la mayoría de los casos por ser su lengua materna: árabe, euskera, japonés, portugués y rumano.

\subsubsection{Nivel acreditado versus resultados obtenidos}

La diferencia entre el nivel acreditado y los resultados obtenidos indica que los estudiantes tienen un nivel certificado más alto del que son capaces de conseguir con esta prueba (vid. Figura 4). Concretamente, en el nivel inferior a un B1 es donde se producen mayores diferencias. En el Listening un $53,86 \%$ obtuvo un nivel inferior a un B1 mientras que tan solo un $28,10 \%$ afirma tenerlo acreditado, existiendo una diferencia del $25,76 \%$. Esa diferencia porcentual es similar en el caso del Reading, donde hay un $46,84 \%$ de estudiantes que han adquirido un nivel inferior al B1, pero tan solo afirman tener acreditado un A2 o A1 un 22,95\%, estableciendo la diferencia porcentual en un 23,89\%. En el resto de los niveles, ese margen de diferencia es algo inferior, pero sigue la tendencia marcada en la que el nivel obtenido en la prueba es inferior que el acreditado.

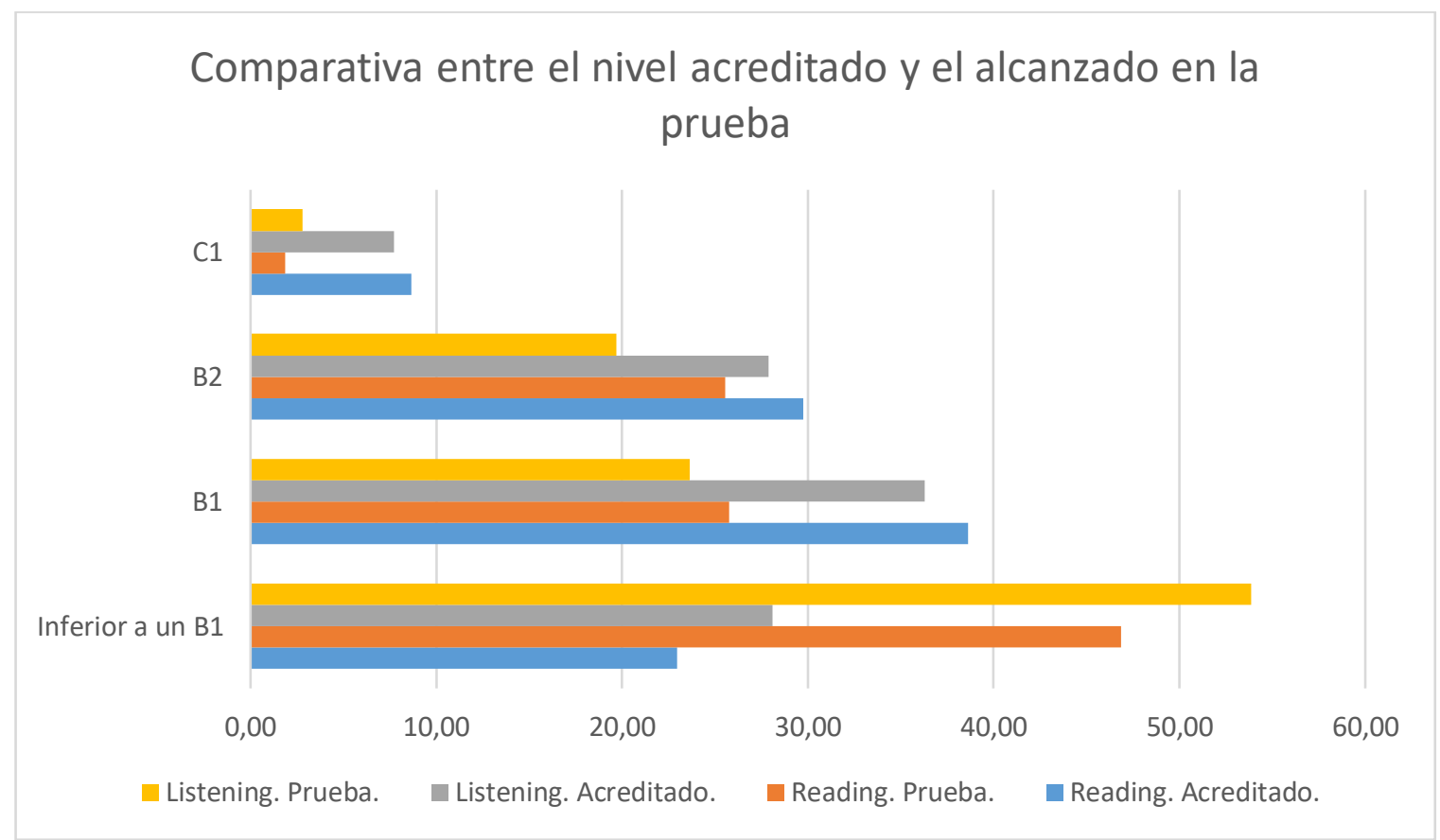

Figura 4. Niveles acreditados versus niveles alcanzados.

Fuente: Elaboración propia.

\subsection{Relación entre la formación en inglés y la competencia lingüística}

\subsubsection{Estudio de inglés}

Para conocer la relación entre los resultados de las pruebas de competencia lingüística en función de la variable estudio de inglés, se han realizado análisis descriptivos considerando tanto el nivel alcanzado en cada prueba como la puntuación. La Figura 5 muestra la distribución de frecuencias por niveles alcanzados según prueba divididos por las dos opciones de la variable estudio de inglés: sí o no. En las tres destrezas analizadas, los informantes que no estudian inglés no han alcanzado en su 
mayoría el B1 necesario para la finalización de sus estudios de Grado mientras que los que más de la mitad de los que sí estudian inglés sí lo han alcanzado: en Reading concretamente un 56,05 \% de los que no estudian inglés obtiene un nivel inferior a B1 mientras que el porcentaje es mucho menor entre los que estudian inglés (36,76\%), en Use of English la distribución es similar $57,40 \%$ frente al $39,22 \%$ y en la prueba de Listening esa distancia parece acortarse ligeramente $60,54 \%$ frente a $46,57 \%$.

Si consideramos los resultados obtenidos de cara a la futura incorporación como docentes de inglés o de enseñanza bilingüe, aproximadamente una cuarta parte de los informantes que estudian inglés consiguen un B2 o superior mientras que el porcentaje en los que no lo estudian es inferior. Concretamente los que estudian inglés han conseguido un $\mathrm{B} 2$ o $\mathrm{C} 1$ en un 33,33\% en Reading, un $25,49 \%$ en Use of English y un 26,96\% en Listening. Por su parte, los que no estudian inglés lo han conseguido en un 21,97 \% en el caso del Reading, un 16,59\% en el Use of English y un 18,39\% en el Listening.

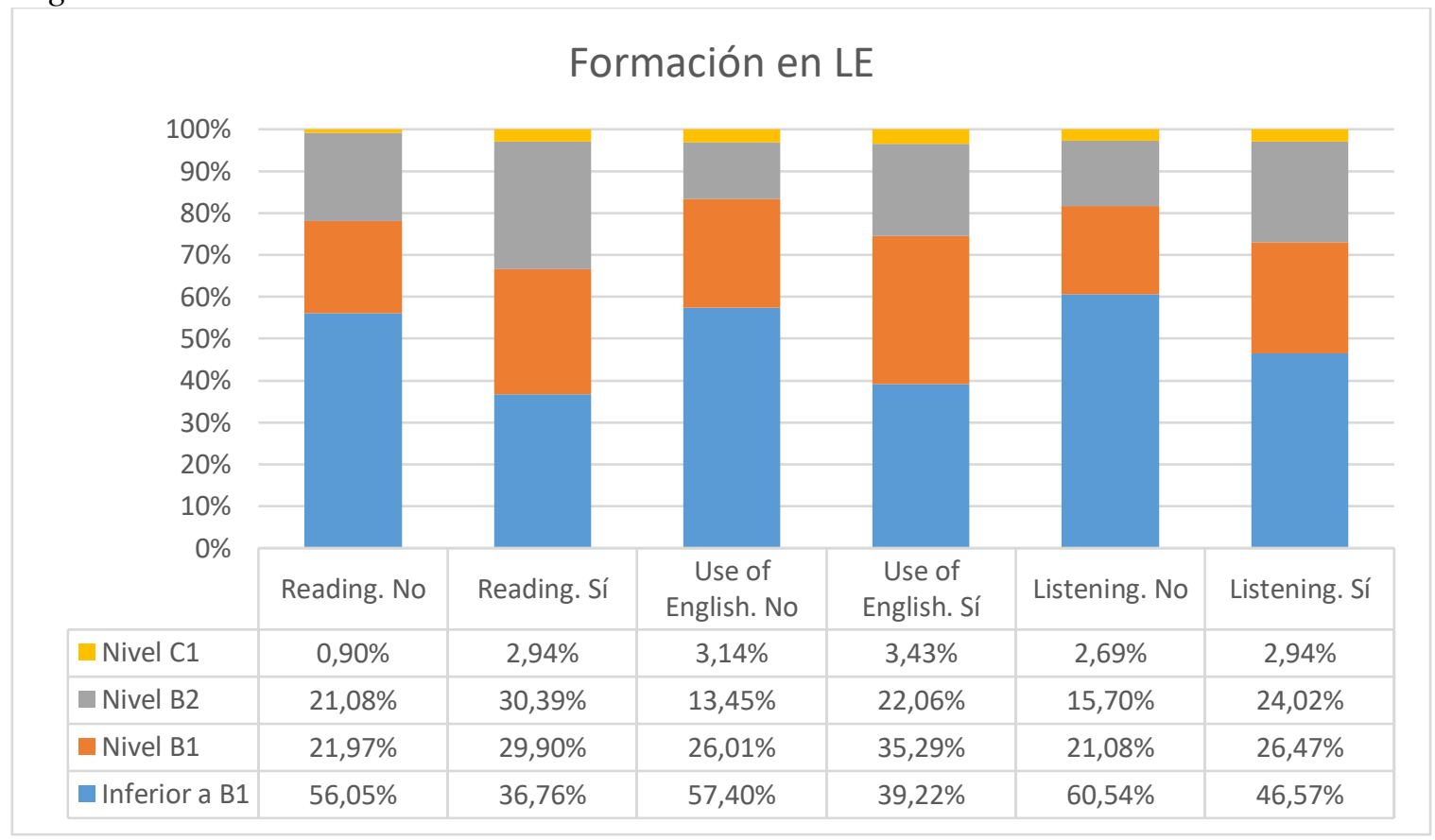

Figura 5. Distribución de los resultados de la prueba de nivel según la variable 'formación'.

Fuente: Elaboración propia.

Con respecto a la puntuación numérica alcanzada, la Tabla 2 corrobora los datos anteriores, ya que la puntuación media de los informantes que estudian inglés es superior en todos los casos al resto mínimo en 2 puntos y supone que los primeros obtengan un nivel equivalente al B1 mientras que los que no estudian inglés tan solo alcancen un nivel A2. En el Reading la media alcanzada por los informantes que estudian inglés es de 18,26 puntos y de los que no estudian inglés es de 15,26; en el Use of English la media de los que estudian inglés es 11,73 y de los que no 9,32. En el Listening 12,41 y 10,37 puntos respectivamente. 
RLCS, Revista Latina de Comunicación Social, 79, 53-75

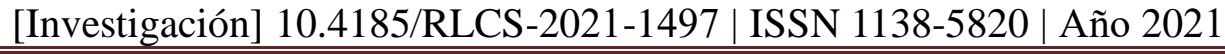

Tabla 2. Estadísticos descriptivos de la puntuación obtenida en las pruebas según la variable estudio de inglés.

\begin{tabular}{|c|c|c|c|c|c|}
\hline \multicolumn{6}{|c|}{ Estadísticas de grupo } \\
\hline & $\begin{array}{l}\text { Estudio de inglés fuera de la } \\
\text { universidad }\end{array}$ & $\mathrm{N}$ & Media & $\begin{array}{l}\text { Desviación } \\
\text { estándar }\end{array}$ & $\begin{array}{l}\text { Media de } \\
\text { error } \\
\text { estándar }\end{array}$ \\
\hline \multirow[t]{2}{*}{ Puntos en el Reading } & No & 223 & 15,26 & 8,661 &, 580 \\
\hline & Sí & 204 & 18,48 & 8,669 & 607 \\
\hline \multirow[t]{2}{*}{ Puntos en el Use of English } & No & 223 & 9,32 & 7,219 & ,483 \\
\hline & Sí & 204 & 11,73 & 6,871 & ,481 \\
\hline \multirow[t]{2}{*}{ Puntos en el Listening } & No & 223 & 10,37 & 6,674 & ,447 \\
\hline & Sí & 204 & 12,41 & 7,051 & 494 \\
\hline
\end{tabular}

Fuente: Elaboración propia.

Para verificar si existen diferencias entre las medias desde un planteamiento estadístico, se ha realizado la prueba t de Student. La Tabla 3 muestra la significación de la $\mathrm{F}$ en la prueba de Levene es $>0,05$, por lo que no se rechaza la hipótesis nula de la igualdad de las varianzas y se asume que las varianzas son iguales. Por ello, se procede con el análisis de la igualdad de medias en el que se observa que el valor de significación de la $t$ es $<0,05$ en todos los casos, por lo que rechazamos la hipótesis nula de la igualdad de medias. En síntesis, se puede concluir que existe una relación significativa entre el estudio de inglés fuera de la universidad y los resultados obtenidos en las pruebas de nivel.

Tabla 3. Prueba T de student de las puntuaciones en las pruebas y el estudio de inglés.

Prueba de muestras independientes

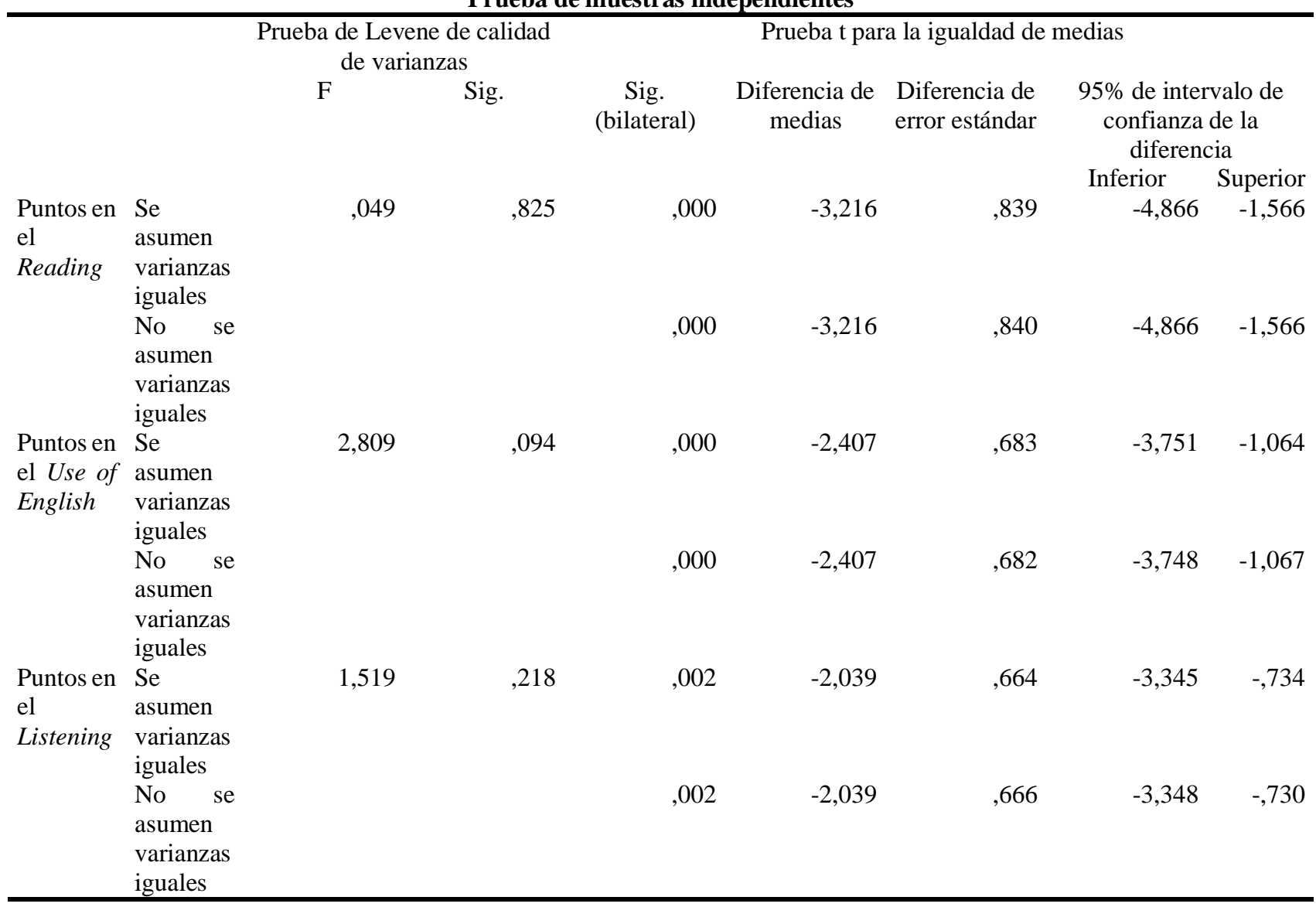

Fuente: Elaboración propia. 


\subsubsection{Estancias en el extranjero}

Siguiendo el mismo procedimiento anterior, la Figura 4 muestra las frecuencias obtenidas en cada uno de los niveles del MCERL según el tipo de prueba y si los informantes han realizado o no estancias en el extranjero. En este caso, se observa que los datos tienden a polarizarse más, de forma que los informantes que han realizado estancias en el extranjero han obtenido en un mayor porcentaje un mínimo de un B1 que el resto. Más de la mitad de los que no han realizado estancias tienen un nivel inferior al B1, concretamente un 50,56\% en el Reading, 51,94\% en el Use of English y 57,78\% en el Listening. En cambio, ese porcentaje es mucho más reducido en los que han realizado estancias: un 26,87\% en el Reading, un 31,34 \% en el Use of English y un 32,84 \% en el Listening.

Si contabilizamos el porcentaje de estudiantes que habría alcanzado al menos un B2, en el caso de los que han realizado estancias supera una cuarta parte: $41,78 \%$ en el Reading, y 32,84 \% tanto en el Use of English como en el Reading. En cambio, el porcentaje es mucho menor en los que no han realizado estancias: 24,72 \% en el Reading, 18,61\% en el Use of English y 20,56\% en el Listening.

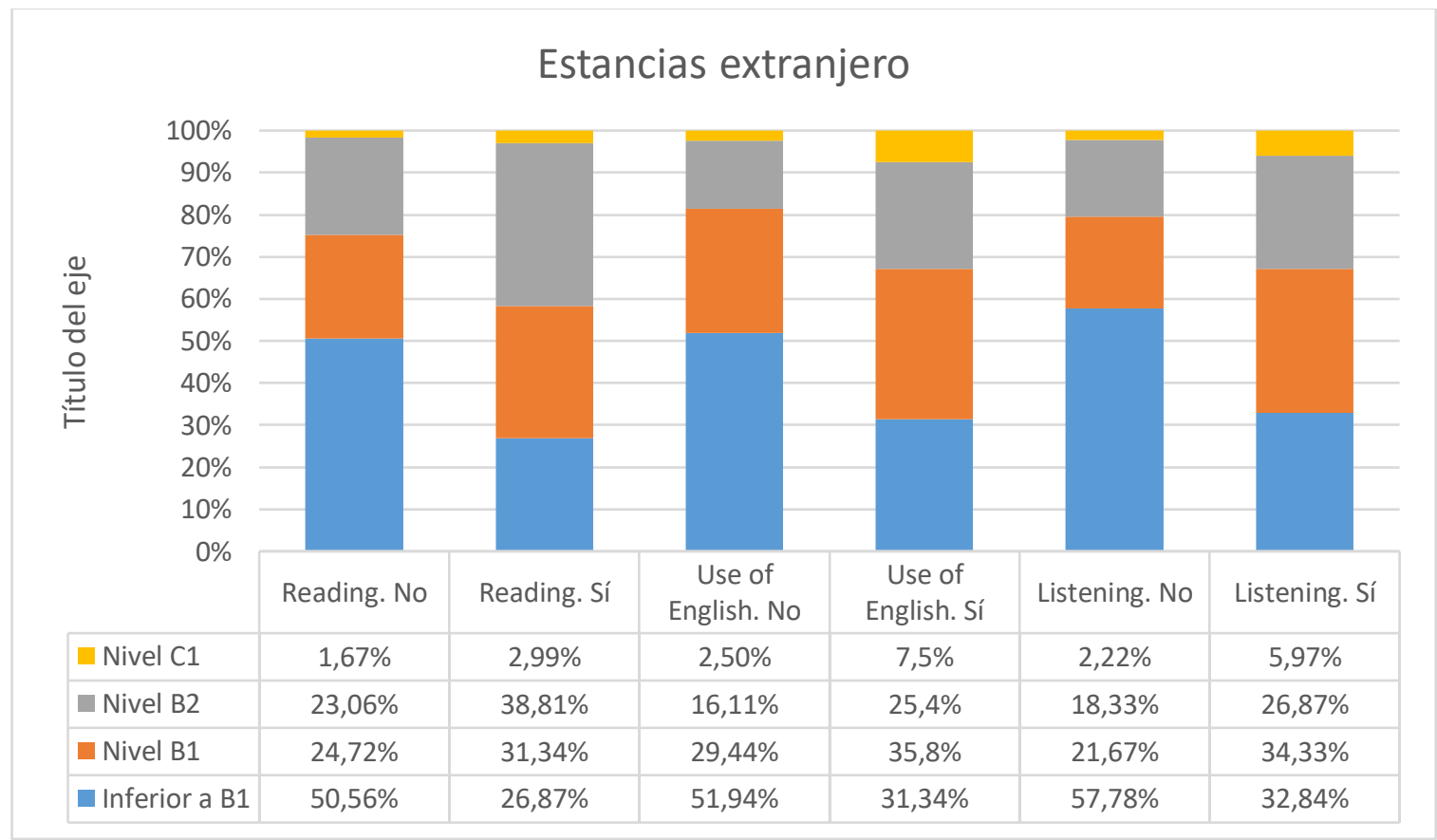

Figura 4. Distribución de los resultados de la prueba de nivel según la realización de estancias en el extranjero.

Fuente: Elaboración propia.

La Tabla 4 presenta los datos descriptivos de las pruebas según la variable estancias en el extranjero. Se observa que los que no hacen estancias han conseguido una puntuación de 16,13 puntos, equivalente al B1 y algo superior que los que no estudiaban inglés (15,26 puntos). Ello se podría deber a que en este grupo se incluyen informantes que sí estudian inglés, pero no han realizado ninguna estancia. En el caso de los que sí hacen estancias, la diferencia con respecto al resto es casi de 4 puntos por encima. En el Reading han obtenido 20,42, en el Use of English 13,45 y en el Listening 14,36 correspondientes al nivel B1. 
RLCS, Revista Latina de Comunicación Social, 79, 53-75

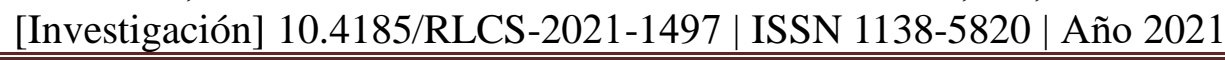

Tabla 4. Estadísticos descriptivos de la puntuación obtenida en las pruebas según la variable "estancia en el extranjero".

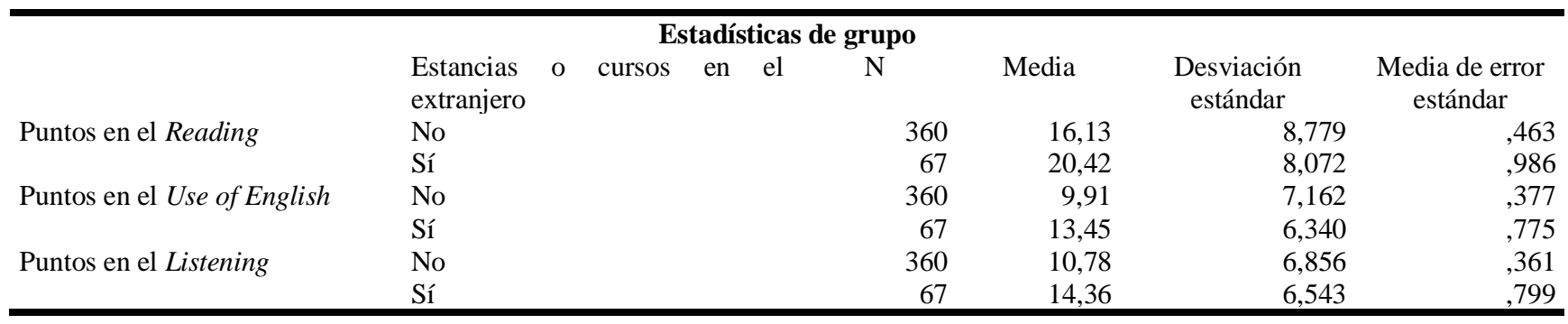

Fuente: Elaboración propia.

La Tabla 5 muestra que existe una relación significativa entre ambas variables. La prueba de Levene rechaza la hipótesis nula de igualdad de varianzas y la prueba t de Student rechaza la hipótesis nula de igualdad de medias. En todos los casos la significación bilateral es inferior a 0,05.

Tabla 5. Prueba $T$ de student de las puntuaciones en las pruebas y las estancias en el extranjero.

Prueba de muestras independientes

\begin{tabular}{|c|c|c|c|c|c|c|c|c|}
\hline & & \multicolumn{2}{|c|}{$\begin{array}{c}\text { Prueba de Levene de calidad } \\
\text { de varianzas }\end{array}$} & \multicolumn{5}{|c|}{ Prueba t para la igualdad de medias } \\
\hline & & \multirow[t]{2}{*}{$\mathrm{F}$} & \multirow[t]{2}{*}{ Sig. } & \multirow[t]{2}{*}{ Sig. (bilateral) } & \multirow[t]{2}{*}{$\begin{array}{l}\text { Diferencia de } \\
\text { medias }\end{array}$} & \multirow[t]{2}{*}{$\begin{array}{l}\text { Diferencia de } \\
\text { error estándar }\end{array}$} & \multicolumn{2}{|c|}{$\begin{array}{l}95 \% \text { de intervalo de } \\
\text { confianza de la } \\
\text { diferencia }\end{array}$} \\
\hline & & & & & & & Inferior & Superior \\
\hline \multirow[t]{2}{*}{$\begin{array}{l}\text { Puntos en } \\
\text { el Reading }\end{array}$} & $\begin{array}{l}\text { Se asumen } \\
\text { varianzas } \\
\text { iguales }\end{array}$ & ,267 & ,606 & , 000 & $-4,290$ & 1,154 & $-6,558$ & $-2,022$ \\
\hline & $\begin{array}{l}\text { No se } \\
\text { asumen } \\
\text { varianzas } \\
\text { iguales }\end{array}$ & & & 000 & $-4,290$ & 1,089 & $-6,452$ & $-2,128$ \\
\hline \multirow[t]{2}{*}{$\begin{array}{l}\text { Puntos en } \\
\text { el Use of } \\
\text { English }\end{array}$} & $\begin{array}{l}\text { Se asumen } \\
\text { varianzas } \\
\text { iguales }\end{array}$ & 3,318 & ,069 & ,000 & $-3,534$ & ,937 & $-5,375$ & $-1,693$ \\
\hline & $\begin{array}{l}\text { No se } \\
\text { asumen } \\
\text { varianzas } \\
\text { iguales }\end{array}$ & & & ,000 & $-3,534$ & ,862 & $-5,243$ & $-1,825$ \\
\hline \multirow[t]{2}{*}{$\begin{array}{l}\text { Puntos en } \\
\text { el } \\
\text { Listening }\end{array}$} & $\begin{array}{l}\text { Se asumen } \\
\text { varianzas } \\
\text { iguales }\end{array}$ & ,382 &, 537 & ,000 & $-3,578$ & ,906 & $-5,358$ & $-1,797$ \\
\hline & $\begin{array}{l}\text { No se } \\
\text { asumen } \\
\text { varianzas } \\
\text { iguales }\end{array}$ & & &, 000 & $-3,578$ &, 877 & $-5,319$ & $-1,836$ \\
\hline
\end{tabular}

Fuente: Elaboración propia.

\section{Discusión}

Al tratarse de alumnado del Grado de Educación, el número de informantes que ha realizado algún tipo de estancia en el extranjero es inferior al de otros estudios realizados en la Universidad de Málaga como el de Santos Díaz (2020), en el marco de estudios de postgrado, donde hay un 63,25 \% de los estudiantes que las ha realizado frente al 15,69\% de este estudio. No obstante, sería interesante analizar 
la movilidad del futuro profesorado en comparación con otros estudios de Grado donde no existen prácticas externas, pues, a pesar de las ventajas detectadas en el trabajo de Sanz Trigueros \& Guillén (2020), aún parece que los futuros maestros ven frenado su deseo de realizar estancias internacionales si no cuentan con experiencias que fomenten un practicum internacional (Sanz-de-la-Cal, 2014).

Cabe mencionar que el nivel diagnosticado en la prueba de inglés es bastante bajo si consideramos que para poder ser docentes en cualquier programa bilingüe o impartir clases de inglés como lengua extranjera necesitarán un B2. Tan solo un 27,40 \% del alumnado tiene un B2 o superior en Reading, un $20,84 \%$ en Use of English y un $22,48 \%$ en Listening. No obstante, si tenemos en cuenta que el objetivo del Plan Estratégico de las Lenguas (Acuerdo de 24 de enero de 2017 de la Junta de Andalucía) es que los docentes que impartan enseñanza bilingüe hayan adquirido al menos un $\mathrm{C} 1$, el porcentaje de futuros docentes que cumpliría este requisito es mínimo, concretamente han alcanzado un $\mathrm{C} 1$ en Reading el 1,87 \%, en Use of English el 3,28 \% y en Listening el 2,81\%. Por tanto, queda patente la necesidad de adecuar la formación inicial del profesorado al contexto actual donde cada vez se requiere más el perfil del docente bilingüe adaptado al marco de la educación superior europea (Guardamillas \& Alcaraz, 2017; Sanz-Trigueros \& Guillén, 2016).

Asimismo, los resultados obtenidos difieren del nivel mínimo que se les presupone al haber acabado los estudios de Bachillerato. La Ley Orgánica para la Mejora de la Calidad Educativa (LOMCE, 2013), reconoce que tras haber superado el bachillerato cada estudiante tiene un nivel de inglés B1 en la primera lengua extranjera cursada. Así, ya desde la promulgación de la Ley Orgánica de Educación (LOE, 2006) se instaba, en el artículo 62.2, a las administraciones educativas a facilitar la realización de pruebas homologadas para obtener la certificación oficial del conocimiento de las lenguas cursadas por los alumnos de Educación Secundaria Obligatoria, Bachillerato y Formación Profesional ${ }^{1}$. No obstante, aunque el nivel depende de muchos factores, tales como, el tipo de centro educativo y los estudios cursados al margen del instituto, al menos se les presupone un nivel B1. Sin embargo, como hemos analizado, casi la mitad de los estudiantes ha obtenido una puntuación inferior al B1 en el Reading y en el Use of English y, en el caso del Listening, supera la mitad.

En cuanto a la disparidad de los resultados obtenidos en las pruebas y los que los estudiantes afirman tener acreditados, habría que analizar qué tipo de exámenes han realizado para acreditar cada uno de los niveles. Es decir: si se trata de aquellas pruebas promovidas por instituciones públicas -Escuelas Oficiales de Idiomas, Asociación de Centros de Lenguas de Enseñanza Superior o Servicios de Idiomas propios de cada universidad- o bien son privadas -APTIS, Trinity, TOEFL o Cambridge- (RaigónRodríguez \& Larrea-Espinar, 2019). Además, sería conveniente indagar acerca del nivel de exigencia planteado en cada uno de los exámenes pues parece extendida la creencia de que existen medios más o menos asequibles para lograr la acreditación idiomática (Ruiz, 2019).

\section{Conclusiones}

A lo largo de estas líneas ha quedado manifiesto que la universidad aún tiene que afrontar un importante desafío: preparar adecuadamente a las generaciones de docentes venideras para socializarse con éxito en una profesión clave para el avance de la sociedad pues, como vienen señalando diversos informes como el realizado por Barber \& Mourshed (2008) para la consultora McKinsey, el nivel educativo de un país depende de la formación, motivación y aprendizaje permanente de su profesorado. Sin embargo, este estudio ha constatado que los futuros maestros no cuentan con un nivel lingüístico adecuado para afrontar los desafíos que plantea la escuela plurilingüe (Abdelillah-Bauer, 2011). Esta cuestión tiene una alta trascendencia para el desarrollo profesional docente pues, sin una adecuada

\footnotetext{
${ }^{1} \mathrm{http} / /$ www.educacionyfp.gob.es/contenidos/estudiantes/ensenanza-idiomas/certificacion.html
} 
competencia lingüística, difícilmente puede alcanzarse una solvencia metodológica desde el enfoque AICLE (Coyle, 2015) ni ejercer una efectiva mediación lectora (Rivera \& Romero, 2020).

Mientras que las administraciones públicas, desde la formación permanente del profesorado (Guardamillas \& Alcaraz, 2017; Guarro et al., 2017) están desarrollando estrategias para que nuestros escolares puedan transitar adecuadamente por la sociedad del conocimiento, parece que la universidad, a pesar de sus esfuerzos por la implantación de grados plurilingües (Delicado \& Pavón, 2015) no está logrando egresar a un profesorado competitivo en este ámbito. Así, se hace inminente una revisión del modelo formativo para lograr desarrollar estrategias que ayuden a la creación de un tercer espacio que permita la colaboración efectiva entre la universidad y la escuela (Zeichner, 2010). pues resultan agentes interdependientes y, como tal, deben actuar con corresponsabilidad.

Al tratarse de una investigación centrada únicamente en la Universidad de Málaga, los resultados no pretenden generalizarse. Sin embargo, los hallazgos encontrados podrían coincidir con los de otros contextos. En este sentido, consideramos necesario emprender trabajos similares que permitan realizar una reflexión conjunta mediante el establecimiento de redes que favorezcan la implementación de proyectos de investigación. De esta forma, se podrán diseñar estrategias conjuntas que ayuden a solventar las deficiencias encontradas.

Como líneas futuras de investigación, se propone la realización de un análisis más pormenorizado de la duración y el tipo de estancias que los estudiantes han realizado en el extranjero. De esta forma, se podrían corroborar los hallazgos de estudios previos (Santos Díaz, 2015; Herranz \& Marcos, 2019) donde los estudiantes que han realizado una estancia en el marco de la beca Erasmus tienen una mayor competencia léxica debido a las características propias de este tipo de becas: tienen una duración de al menos tres meses y los estudiantes suelen adquirir la lengua extranjera tanto en un contexto natural como académico. Además, incluyendo el componente cultural, se podría seguir profundizando en la línea de trabajo trazada por Šifrar (2020).

Asimismo, aparte de que reciban clases de idiomas y cultura, sobre todo en el primer mes de acogida, los contenidos se dan en lengua extranjera y, por tanto, se podría decir que adquieren la lengua a través de la metodología del Aprendizaje Integrado de Contenidos en Lengua Extranjera (AICLE), enfoque que habrán de utilizar en el aula al ejercer su profesión como docentes activos en el marco de la educación plurilingüe (Fernández-Viciana \& Fernández-Costales, 2019; Martín-Macho \& Faya, 2020; Otto \& Estrada, 2019).

Unido a esto, sería interesante conocer cuál es la exposición a la lengua extranjera de los informantes ya que se ha mostrado en estudios anteriores que puede ser de gran relevancia tanto si se trata del uso de la lengua en el ámbito académico (Santos Díaz, 2018), a través de sus lecturas en lengua extranjera (Fernández Ruiz et ál., 2018; Gómez López, 2014) como de la exposición informal a través de amigos y familiares (Santos Díaz et ál., 2020) o de formas de ocio relacionadas con el uso de internet o plataformas digitales (Shafirova et ál., 2020; Zhang \& Cassany, 2019).

Finalmente, pasados unos años, debería evaluarse cómo ha influido la pandemia declarada por la COVID-19 en la enseñanza universitaria, en general (Tejedor, et ál., 2020) y en la de idiomas, en particular (Ruiz-Zamora, 2020) pues es indudable que la tecnología jugará un papel protagonista en los modos de comunicación entre docentes y discentes (Álvarez \& Alejaldre, 2019; Barrientos et ál., 2020). 


\section{Referencias}

Abdelillah-Bauer, B. (2011). El desafío del bilingüismo. Morata.

Acuerdo de 22 de marzo de 2005, del Consejo de Gobierno, por el que se aprueba el Plan de Fomento del Plurilingüismo en Andalucía. Boletín Oficial de la Junta de Andalucía, núm. 65, de 5 de abril de 2005, pp. 8-39. http://www.juntadeandalucia.es/boja/2005/65/d5.pdf

Acuerdo de 24 de enero de 2017, del Consejo de Gobierno, por el que se aprueba el Plan Estratégico de Desarrollo de las Lenguas en Andalucía Horizonte 2020. Boletín Oficial de la Junta de Andalucía, núm. 24, de 6 de febrero de 2017, pp. 10-57. http://www.juntadeandalucia.es/boja/2017/24/BOJA17-024-00048-1794-01 00107169.pdf

Aguilar, S. \& Barroso, J. (2015). La triangulación de datos como estrategia en investigación educativa. $\begin{array}{llllll}\text { Píxel-Bit. Revista de Medios } & y & \text { Educación, } & 47, & 73-88 .\end{array}$ http://dx.doi.org/10.12795/pixelbit.2015.i47.05

Álvarez, E. \& Alejaldre, L. (2019). Las TIC como mediadoras en el aprendizaje de lenguas extranjeras en Educación Infantil. Aproximación desde el enfoque natural. Tejuelo, 30, 175-206. https://doi.org/10.17398/1988-8430.30.175

Barrientos, A., Caldevilla, D. \& Rodríguez, J. (2020). Comunicación y enseñanza a través de las plataformas "on line": una lectura diferente. En A. E. Díez-Mediavilla \& R. Gutiérrez (Coord.), Lectura y dificultades lectoras en el siglo XXI (pp. 49-60). Octaedro.

Barber, M. \& Mourshed, M. (2008). Cómo hicieron los sistemas educativos con mayor desempeño del mundo para alcanzar sus objetivos. Mckinsey \& Company.

Cabero, J. \& Llorente, M. C. (2013). La aplicación del juicio de experto como técnica de evaluación de las tecnologías de la información (TIC). Eduweb. Revista de Tecnología de la Información y la Comunicación, 7(2), 11-22.

Castillo, C., Díaz, J. M. \& Rubio, B. (2020). Compiling and analyzing a tagged learner corpus: a corpus-based study of adjective uses. Círculo de Lingüística Aplicada a la Comunicación, 81, 115136, http://dx.doi.org/10.5209/CLAC.67932

Choudaha, R. (2014). Challenges and opportunities for Global student mobility in the future: A comparative and critical analysis. En B. Streitwieser (Coord.), Internationalization of Higher Education and Global Mobility (pp. 19-33). Symposium Book.

Consejo de Europa (2001). Marco común europeo de referencia para las lenguas: aprendizaje, enseñanza, evaluación. Traducido por el Instituto Cervantes. Anaya.

Cordero, J. M. \& Gil-Izquierdo, M. (2018). The effect of teaching strategies on student achievement: An analysis using TALIS-PISA-link. Journal of Policy Modeling, 40(6), 1313-1331. https://doi.org/10.1016/j.jpolmod.2018.04.003

Coyle, D. (2015). Strengthening integrated learning: towards a new era for pluriliteracies and intercultural learning. Latin American Journal of Content and Language Integrated Learning, 8(2), 84-103. https://doi.org/10.5294/laclil.2015.8.2.2 
Delicado, G. \& Pavón, V. (2015). La implantación de titulaciones bilingües en la educación superior: el caso de la formación didáctica del profesorado bilingüe de primaria en la Universidad de Extremadura. Educación y Futuro, 32, 35-63.

Escobar, J. \& Cuervo, A. (2008). Validez de contenido y juicio de expertos: una aproximación a su utilización. Avances en medición, 6, 27-36. https://www.researchgate.net/publication/302438451

Estrada, J. L. \& Zayas, F. (2019). Creencias de futuros docentes sobre la gramática en la enseñanza de idiomas. Enunciación, 24(2), 152-168. http://doi.org/10.14483/22486798.14838

Fernández-Ruiz, M. R., Corpas, G. \& Seghiri, M. (2018). Recepción en España de la literatura africana en lengua inglesa: generación de datos estadísticos con la base de datos bibliográfica especializada BDÁFRICA. Artnodes: revista de arte, ciencia y tecnología, 22, 50-61. http://dx.doi.org/10.7238/a.v0i22.3210

Fernández-Viciana, A. \& Fernández-Costales, A. (2019). La autoeficacia percibida en los futuros docentes de inglés de Educación Primaria. Profesorado. Revista currículum y formación de profesorado, 23(4), 216-233. https://doi.org/10.30827/profesorado.v23i4.11719

Forteza, A., De Casas, P. \& Vizcaíno, A. (2020). El grado de alfabetización digital en el profesorado de inglés en Educación Primaria. International Journal of Educational Research and Innovation, 14, 76-90. https://doi.org/10.46661/ijeri.4038

Gómez López, Á. (2014). Los hábitos lectores en inglés de futuros maestros: implicaciones didácticas. Investigaciones Sobre Lectura, 2, 24-31. https://doi.org/10.37132/isl.v0i2.3

González-Peiteado, M. (2013). Los estilos de enseñanza y aprendizaje como soporte de la actividad docente. Revista de estilos de aprendizaje, $11, \quad$ 51-70. http://revistaestilosdeaprendizaje.com/article/view/971

Guardamillas, M. V. \& Alcaraz, G. (2017). Legislación en enseñanza bilingüe: Análisis en el marco de Educación Primaria en España. Multiárea. Revista de didáctica, 9, 82-103. https://doi.org/10.18239/mard.v0i9.1528

Guarro, A., Martínez, B. \& Portela, A. (2017). Políticas de formación continuada del profesorado: análisis crítico del discurso oficial de Comunidades Autónomas._Profesorado. Revista currículum y formación de profesorado, 21(3), 21-40.

Herranz, C. V. \& Marcos, M. Á. (2019). Análisis del léxico disponible español de extranjeros que estudian los grados de educación. Ogigia. Revista electrónica de estudios hispánicos, 26, 5-30. https://doi.org/10.24197/ogigia/26.2019.6-30

Lauridesen, K. M. \& Lilemose, M. K. (2015) Opportunities and challenges in the multilingual and multicultural learning space. Final document of the IntlUni Erasmus Academic Network project 2012- 15. https://doi.org/10.13140/RG.2.1.1917.9929

Lorenzo, F. (2016): Competencia en comunicación lingüística: claves para el avance de la comprensión lectora en las pruebas PISA. Revista de Educación, 374, 142-160. https://doi.org/10.4438/1988592X-RE-2016-374-329 
Lorenzo, F. \& Rodríguez, L. (2014). Onset and expansions of L2 cognitive academic language proficiency in bilingual settings: CALP in CLIL. System, 47, 64-72. https://doi.org/10.1016/j.system.2014.09.016

Martín del Pozo, M. Á. M. (2013). Formación del profesorado universitario para la docencia en inglés. REDU. Revista de Docencia Universitaria, 11(3), 197-208. https://doi.org/10.4995/redu.2013.5526

Martín-Macho, A. \& Faya, F. (2020). L1 in CLIL: the case of Castilla-La Mancha. Tejuelo, 31, 143174. https://doi.org/10.17398/1988-8430.31.143

Martín-Izard, J. F. (2010). Técnicas de encuesta: cuestionario y entrevista. En S. Nieto (Coord.), Principios, Métodos y Técnicas esenciales para la Investigación educativa (pp. 145-168). Dykinson.

Martínez-Agudo, J. L. (2011). Perfil profesional idóneo del profesor de lengua extranjera: creencias del profesorado en formación. Revista Latinoamericana de estudios educativos, XLI(1-2), 103-124. https://www.redalyc.org/articulo.oa?id=27021144004

Matas, A. (2018). Diseño del formato de escala Likert: el estado de la encuesta. Revista Electrónica de Investigación Educativa, 20(1), 38-47. https://doi.org/10.24320/redie.2018.20.1.1347

McMillan, J. H. \& Schumacher, S. (2006). Investigación educativa. (5a Ed.). Pearson.

Meyer, O., Coyle, D., Halbach, A., Schuck, K. \& Ting, T. (2015). A pluriliteracies approach to content and language integrated learning - mapping learner progressions in knowledge construction and meaning-making. Language, Culture and Curriculum, 28(1), 41-57. https://doi.org/10.1080/07908318.2014.1000924 .

Ministerio de Educación, Cultura y Deporte (2006). Ley Orgánica 2/2006, de 3 de mayo, de Educación.

Ministerio de Educación, Cultura y Deporte (2013). Ley Orgánica de 9 de diciembre para la Mejora de la Calidad Educativa.

Mottareale, D. (2017). La internacionalización de la docencia en educación superior: el caso de la Universidad Complutense [Tesis inédita de doctorado, Universidad Complutense de Madrid]. Repositorio institucional. https://eprints.ucm.es/48347/1/T40331.pdf

Otto, A. \& Estrada, J. L. (2019). Towards an Understanding of CLIL in a European Context: Main Assessment Tools and the Role of Language in Content Subjects. CLIL Journal of Innovation and Research in Plurilingual and Pluricultural Education, 2(1), 31-42. https://doi.org/10.5565/rev/clil.11

Páez, V. (2013). El profesor de idiomas: sus cualidades y competencias. Revista Comunicación, 11(3). https://doi.org/10.18845/rc.v11i3.1265

Pavón, V. \& Pérez Invernón, A. (2018). Enhancing disciplinar literacies: languages of schooling and whole-school language projects in Spain. European Journal of Applied Linguistics, 6(1), 109-130. https://doi.org/10.1515/eujal-2017-0023 
RLCS, Revista Latina de Comunicación Social, 79, 53-75

\begin{tabular}{ll|l|l|l} 
[Investigación] 10.4185/RLCS-2021-1497 & ISSN 1138-5820 & Año 2021
\end{tabular}

Pérez Invernón, A. (2019). El Proyecto Lingüístico de Centro: una evolución necesaria para la mejora de la Competencia en Comunicación Lingüística. Tejuelo, 30, 13-36. https://doi.org/10.17398/1988-8430.30.13

Pérez Invernón, A., Lorenzo, F. \& Pavón, V. (2016). European bilingual models beyond lingua franca. Key findings from CLIL French programs. Language Policy, 15(4), 485-504. https://doi.org/10.1007/s10993-015-9386-7

Pérez-Parejo, R., Gutiérrez-Cabezas, Á., Soto-Vázquez, J., Jaraíz-Cabanillas, F. J. \& GutiérrezGallego, J. A. (2018). Hábitos de lectura en lenguas extranjeras en los estudiantes de Extremadura. Ocnos: $\quad$ Revista De Estudios $\quad$ Sobre Lectura, 17(2), 67-81. https://doi.org/10.18239/ocnos 2018.17.2.1714

Raigón-Rodríguez, A. \& Larrea-Espinar, Á. (2019). La acreditación de idiomas en las universidades españolas: Un estudio cuantitativo en la Universidad de Córdoba. Journal of Educational Research and Innovation, 12, 245-260.

Rivera, P. \& Romero, M. F. (2020). El adolescente como lector accidental de textos literarios: hábitos de lectura literaria en Educación Secundaria. Ocnos: Revista De Estudios Sobre Lectura, 19(3). https://doi.org/10.18239/ocnos_2020.19.3.2313

Robles, P. \& Rojas, M. C. (2015). La validación por juicio de expertos: dos investigaciones cualitativas en Lingüística aplicada. Revista Nebrija de Lingüística aplicada, 18. https://www.nebrija.com/revista-linguistica/files/articulosPDF/articulo_55002aca89c37.pdf

Ruiz, M. B. (2019). La acreditación del nivel de lengua inglesa en la Universidad de Castilla-La Mancha. Multiárea. Revista de didáctica, 10, 60-85. https://doi.org/10.18239/mard.v0i10.2092

Ruiz-Zamora, U. (2020). Competencia digital de estudiantes universitarios para el aprendizaje del inglés en tiempos de la COVID-19. Revista Lengua y Cultura, 2(3), 102-109. https://doi.org/10.29057/lc.v2i3.6575

Sáez, J. (2017). Investigación educativa. Fundamentos teóricos, procesos y elementos prácticos (enfoque práctico con ejemplos. Esencial para TFG, TFM y tesis). UNED.

Santos Díaz, I. C. (2015). El impacto de las estancias en el extranjero en el léxico disponible en inglés y francés de estudiantes de postgrado. e-AESLA, 1 . https://cvc.cervantes.es/lengua/eaesla/pdf/01/32.pdf

Santos Díaz, I. C. (2018) Incidencia del uso de la lengua extranjera (inglés y francés) en clase sobre el léxico disponible. $e$-AESLA, 4, 95-100. https://cvc.cervantes.es/ lengua/eaesla/pdf/04/09.pdf

Santos Díaz I. C., Trigo, E. \& Romero, M. F. (2020). Relación entre la exposición informal a una lengua extranjera (inglés y francés) y la competencia léxica. En I. Karapetjana (Coord.), Language for International Communication: Linking Interdisciplinary Perspectives (pp. 431-439). University of Latvia Press. https://doi.org/10.22364/lincs.2020.38

Sanz de la Cal, E. (2014). La movilidad en la formación inicial de maestros en lenguas extranjeras: el caso de un Prácticum Internacional [Tesis inédita de doctorado, Universidad de Valladolid]. Repositorio institucional. https://uvadoc.uva.es/handle/10324/4973 
Sanz Trigueros, F. J. \& Guillén, C. (2016). La autoeficacia percibida del docente de Lenguas Extranjeras, desde un dispositivo estratégico y operativo de identificación de su profesionalización y desarrollo profesional. Porta Linguarum: revista internacional de enseñanza de lenguas extranjeras, 26, 93-107. https://doi.org/10.30827/Digibug.53927

Sanz Trigueros, F. J. \& Guillén, C. (2020). La movilidad temporal internacional. Aproximación a sus efectos en el desarrollo profesional de docentes de y en lenguas extranjeras. Bordón. Revista de pedagogía, 73(1), 133-150. https://doi.org/10.13042/Bordon.2020.01.72054

Shafirova, L., Cassany, D. \& Bach, C. (2020). From "newbie" to professional: Identity building and literacies in an online affinity space. Learning, Culture and Social Interaction, 24, 100370. https://doi.org/10.1016/j.lcsi.2019.100370

Šifrar, M. (2020). Estereotipos culturales sobre España en estudiantes universitarios extranjeros: el caso de los Erasmus eslovenos. Ogigia. Revista electrónica de estudios hispánicos, 27, 213-234. https://doi.org/10.24197/ogigia.27.2020.213-234

Sologuren, E. \& Castillo-Fadić, M. N. (2020). Reading in and across disciplines: comprehension of written academic texts. Exploring the field from some comprehension theories and models of written discourse. Investigaciones Sobre Lectura, 14, 42- 57. https://doi.org/10.37132/isl.v0i14.320

Tejedor, S., Cervi, L., Tusa, F. \& Parola, A. (2020). Educación en tiempos de pandemia: reflexiones de alumnos y profesores sobre la enseñanza virtual universitaria en España, Italia y Ecuador. Revista Latina de Comunicación Social, 78,1-21. https://doi.org/10.4185/RLCS-2020-1466

Torrado-Crespón, M. (2018). Interlanguage or Technology when Using English as Vehicular Language: what influences students productions online? The EuroCALL Review, 26(2), 41-49. https://doi.org/10.4995/eurocall.2018.9924

Trigo, E., Romero, M. F. \& García, Á. (2019). Las voces de los agentes dinamizadores en la implementación de un PLC como clave para la transformación de un centro educativo. Tejuelo, 30, 37-72. https://doi.org/10.17398/1988-8430.30.37

Yance, R. L., Beltrán, D. M. I. \& Alfonso, G. A. (2011). El profesor de idioma inglés de excelencia. Revista Cubana de Educación Médica Superior, 25(2), 178-185. http://scielo.sld.cu/pdf/ems/v25n2/ems14211.pdf

Wulz, J. (2015). Challenges of student mobility in a Cosmopolitan Europe. En A. Curaj, L. Matei, R. Pricopie, J. Salmi \& P. Scott (Coords.), The European Higher Education (pp. 43-58). Springer.

Zeichner, K. M. (2010). Nuevas epistemologías en formación del profesorado. Repensando las conexiones entre las asignaturas del campus y las experiencias de prácticas en la formación del profesorado en la universidad. Revista Interuniversitaria de Formación del Profesorado, 68, 123149. https://www.redalyc.org/articulo.oa?id=27419198007

Zhang, L. T. \& Cassany, D. (2019). Prácticas de comprensión audiovisual y traducción en una comunidad fansub del español al chino. Revista Española de Lingüística Aplicada, 32(2), 620649. https://doi.org/10.1075/resla.17013.zha 


\section{AUTORAS:}

\section{Ester Trigo Ibáñez}

Doctora en Lingüística por la Universidad de Cádiz (2007). Profesora Contratada Doctora en el Departamento de Didáctica de la Lengua y la Literatura de la Universidad de Cádiz. Sus líneas de investigación son diversas: escritura académica, desde la que ha publicado varios capítulos en altos niveles de SPI; educación literaria, desde donde ha dirigido una tesis doctoral y publicado varios artículos SJR; disponibilidad léxica, cuya tesis doctoral forma parte del Proyecto Panhispánico de Disponibilidad Léxica, desde esta línea ha publicado varios trabajos en JCR y SJR y proyectos lingüísticos de centro, desde la que ha conectado con el profesorado en activo. Sus publicaciones son resultado de: la participación en proyectos de investigación, de acciones avaladas para la mejora docente, de tesis doctorales dirigidas, de su propia tesis o de estancias de investigación en Polonia, Chile y Portugal.

ester.trigo@uca.es

\section{Índice H: 10}

Orcid ID: https://orcid.org/0000-0003-3035-4398

Google Scholar: https://scholar.google.com/citations?hl=es\&user=vf4WkFwAAAAJ

ResearchGate: https://www.researchgate.net/profile/Ester Trigo-Ibanez

Scopus ID: https://www.scopus.com/authid/detail.uri?authorId=57202802502

Academia.edu: https://circulodelestrecho.academia.edu/EsterTrigoIb\%C3\%A1\%C3\%B1ez

\section{Inmaculada Clotilde Santos Díaz}

Doctora en Innovación Educativa por la universidad de Málaga (2015). Es Profesora Ayudante Doctora en el Departamento de Didáctica de la Lengua, las Artes y el Deporte en la Universidad de Málaga y evaluadora experta y controladora de proyectos Erasmus + para el Servicio Español para la Internacionalización de la Educación. Tiene una amplia experiencia en la gestión educativa como Coordinadora Provincial de Lectura y Biblioteca Escolares de la Delegación Territorial de Educación de Málaga Su investigación, transferida en revistas JCR y SJR se centra en la lingüística aplicada a la enseñanza de idiomas donde cuenta con una amplia formación. Actualmente, forma parte de dos proyectos europeos y es la coordinadora académica de las movilidades KA203 con China. Asimismo, ha participado en numerosos congresos internacionales.

santosdiaz@uma.es

Índice H: 6

Orcid ID: https://orcid.org/0000-0002-0066-7783

Google Scholar: https://scholar.google.com/citations?user=MT-CezAAAAAJ\&hl=es

ResearchGate: https://www.researchgate.net/profile/Inmaculada_Santos_Diaz

Scopus ID: https://www.scopus.com/authid/detail.uri?authorId=57194426317

Academia.edu: https://uma.academia.edu/InmaSantos

\section{Guillermina Jiménez López}

Doctoranda del programa del doctorado de Lingüística, Literatura y Traducción. Es profesora interina en el Departamento de Didáctica de las Lenguas, las Artes y el Deporte en la Universidad de Málaga. Con anterioridad, desarrolló una prolongada labor de enseñanza del inglés como lengua extranjera en diferentes programas de Formación profesional para el empleo de la Junta de Andalucía. Su investigación se centra en la formación en lengua extranjera del futuro profesorado de Educación Primaria. La cual se está publicando como capítulos de libro en editoriales de reconocido prestigio como Dyckinson, Síntesis o Gedisa. En la actualidad, forma parte de un proyecto de investigación sobre la incorporación de la técnica break-out educativo al ámbito universitario.

gjimenez@uma.es 
RLCS, Revista Latina de Comunicación Social, 79, 53-75

\begin{tabular}{ll|l|l|l} 
[Investigación] 10.4185/RLCS-2021-1497 & ISSN 1138-5820 & Año 2021
\end{tabular}

Orcid ID: https://orcid.org/0000-0001-6341-7637

ResearchGate: https://www.researchgate.net/profile/G_Jimenez2 